\title{
${ }^{\curvearrowright}$ A Diurnal Predictability Barrier for Weather Forecasts
}

\author{
Peng Wang, ${ }^{\text {a }}$ Yishuai Jin, ${ }^{\text {b,c }}$ AND Zhengyu LiU ${ }^{\mathrm{d}}$ \\ ${ }^{a}$ Department of Atmospheric and Oceanic Sciences, Peking University, Beijing, China \\ ${ }^{\mathrm{b}}$ Key Laboratory of Physical Oceanography, Ministry of Education, Ocean University of China, Qingdao, China \\ ${ }^{\mathrm{c}}$ Open Studio for Ocean-Climate-Isotope Modeling, Pilot National Laboratory for Marine Science and \\ Technology (Qingdao), Qingdao, China \\ d Atmospheric Science Program, Department of Geography, The Ohio State University, Columbus, Ohio
}

(Manuscript received 22 June 2020, in final form 9 March 2021)

\begin{abstract}
In this study, we investigate a diurnal predictability barrier (DPB) for weather predictions using an idealized model and observations. This DPB is referred to a maximum drop of predictability (e.g., autocorrelation) at a particular time of the day, regardless of the initial time. Previous studies demonstrated that a strong seasonal cycle of El NiñoSouthern Oscillation (ENSO) growth rate is responsible for the seasonal predictability barrier of the ENSO in spring. This led us to investigate whether or not a strong diurnal cycle may generate a DPB. We study the DPB using an idealized model, the Lorenz 1963 model, with the addition of a diurnal cycle. We find that diurnal growth rate can generate a DPB in this chaotic system, regardless of the initial error. Finally, by calculating the autocorrelation function using the hourly data of surface temperature, we explore the DPB at two stations in Wisconsin and Beijing, China. A clear DPB feature is found at both stations. The dramatic drop of predictability at a specific time of the day is likely due to the diurnal variation of the system. This is a new feature that needs further study for short-term weather predictions.
\end{abstract}

KEYWORDS: Automatic weather stations; Climate prediction; Forecast verification/skill; Numerical weather prediction/forecasting; Statistical forecasting

\section{Introduction}

One important feature of El Niño-Southern Oscillation (ENSO) prediction is the seasonal predictability barrier in spring. It has long been observed in the tropical Pacific $\left(5^{\circ} \mathrm{S}-\right.$ $5^{\circ} \mathrm{N}, 170^{\circ}-120^{\circ} \mathrm{W}$ ) sea surface temperature anomaly (SSTA) variability (e.g., Niño-3.4; Torrence and Webster 1998; Ren et al. 2016), and the variability in sea level pressure (Troup 1965; Webster and Yang 1992) and rainfall (Walker and Bliss 1932; Wright 1979). The spring predictability barrier is characterized by a maximum decline in the autocorrelation function (ACF) of monthly SSTA (e.g., Liu et al. 2019) and other monthly parameters, such as rainfall (Walker and Bliss 1932; Wright 1979), in the tropical Pacific. This seasonal phaselocking of spring predictability barrier indicates that, regardless of the initial month, a damped forecast loses its predictability most rapidly in the following May or June, forming the spring predictability barrier of the ENSO (e.g., Ren et al., 2016).

Recently, through the Langevin equation (Hasselmann 1976) with a seasonal cycle in its growth rate or noise forcing, Liu et al. (2019) showed theoretically that the seasonal predictability barrier is an intrinsic feature of a linear, stochastic climate system as long as the seasonal forcing is sufficiently strong. More specifically, their approximate analytical solution

\footnotetext{
D Denotes content that is immediately available upon publication as open access.
}

Corresponding author: Yishuai Jin, jinyishuai@126.com; ZhengyuLiu,liu.7022@osu.edu indicated that a predictability barrier always exists when the periodic forcing is strong enough. Therefore, the well-known spring predictability barrier is caused by the seasonally varying growth rate of ENSO SSTA (Levine and McPhaden 2015; Liu et al. 2019). This led us to speculate that a diurnal predictability barrier (DPB) may be present for daily weather variability, because of the presence of a prominent diurnal cycle worldwide. According to the definition of ENSO spring predictability barrier, a DPB indicates that, regardless of the initial hour, the predictability will exhibit a maximum drop at a particular hour of the day. It explains a drop of predictability at a specific hour of the day due to the diurnal cycle of the weather system (more details about this null hypothesis are given in the appendix).

However, as the development of a weather system tends to be nonlinear and chaotic (Orrell 2002), it is not yet clear if the seasonal predictability barrier theory derived from the linear, damped model can be applied to a chaotic weather system. Therefore, we will test the theory derived from the linear, damped model (Liu et al. 2019) in a DPB using a prototypical model of convection, namely, the Lorenz 1963 model (Lorenz 1963, Lorenz63 model hereafter). We will show that incorporating a diurnal cycle in the Lorenz63 model can generate a strong DPB. We then confirm the presence of such DPB in the real world by calculating the ACF of surface temperatures at two stations in Wisconsin and Beijing, China.

This paper is arranged as follows. The station data, models, and the definition of DPB strength used in this paper are introduced in section 2. We study the DPB in the diurnal forcing of the Lorenz63 model in section 3. The DPB observed from surface station data is introduced in section 4. A summary and discussion are given in section 5 . 

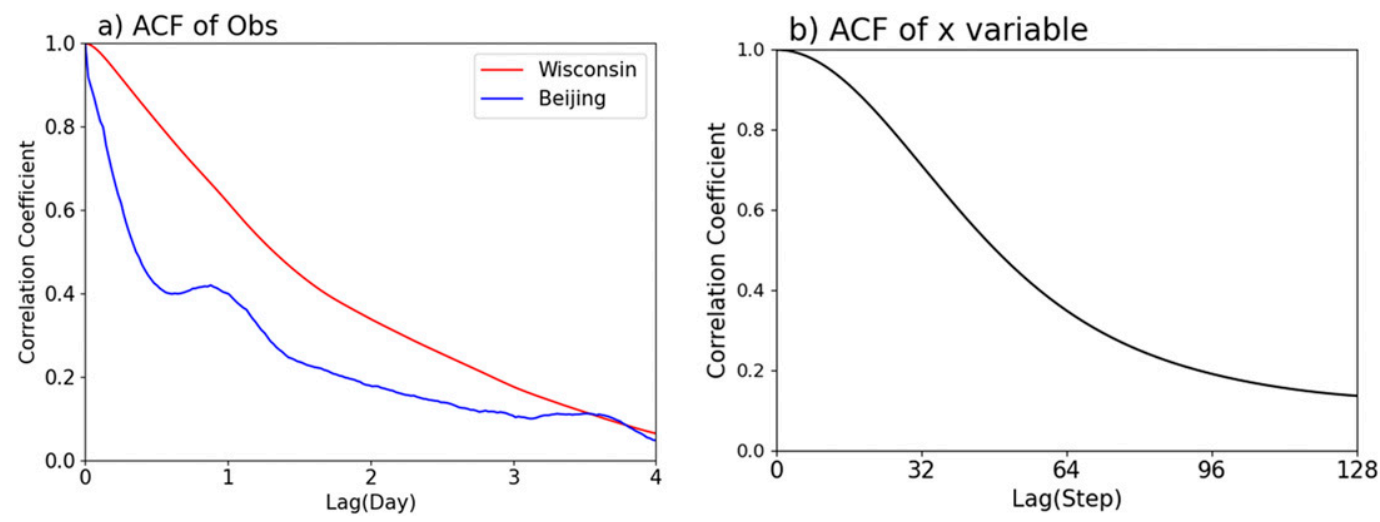

FIG. 1. Lag correlation of (a) observed surface temperatures in Wisconsin (red line) and Beijing (blue line) in January and (b) variable $x$ in Lorenz63 model $(A=0)$.

\section{Methodology}

\section{a. Data}

Two sets of 30-min station data are used to study the DPB in the real world. One set is Wisconsin $\left(45.8^{\circ} \mathrm{N}, 90.0^{\circ} \mathrm{W}\right)$ station data (from 1998 to 2018) from Ankur Desai's Ecometeorology Laboratory of University of Wisconsin-Madison (http://co2.aos.wisc.edu/data/cheas/wcreek/flux/current/). The other is Beijing Capital International Airport station $\left(40.0^{\circ} \mathrm{N}\right.$, $116.5^{\circ} \mathrm{E}$; Weather Station Identifier: 54511) data (from 2009 to 2018) from NOAA's National Centers for Environmental Information (NCEI) (https://www.ncei.noaa.gov/data/globalhourly/access/). Note that 30-min resolution station data are not available before 2009, which is why data after 2009 are used in this paper to investigate the DPB. All of the 30-min datasets used here are preprocessed. We choose the surface temperature time series of Wisconsin and Beijing in January and July, respectively, to check the DPB in the real world (more details can be found in section 2e). For a specific calendar month from 1998 to 2018 for Wisconsin station data and from 2009 to 2018 for Beijing station data, the linear trend can be calculated and removed. Similarly, the diurnal climatology can be calculated and removed.

\section{b. Lorenz63 model}

The Lorenz63 model with an added diurnal cycle component can be written as follows:

$$
\begin{aligned}
\frac{d x}{d t} & =\alpha y-\alpha \times[1-A \cos (\theta)] x, \\
\frac{d y}{d t} & =\gamma x-y-x z, \\
\frac{d z}{d t} & =-b z+x y, \\
\theta & =2 \pi \frac{t}{T},
\end{aligned}
$$

where $x, y$, and $z$ are the three variables in the system. It describes one of the most famous nonlinear dissipative dynamical systems (Palmer 1993). The parameters $\alpha=10, \gamma=28$, and $b=8 / 3$ are set in the same way as in the classical Lorenz63 model. A diurnal cycle $(\theta=2 \pi(t / T))$ is added to this system as part of the coefficient of Eq. (2.1), namely, $-\alpha \times[1-A \cos (\theta)]$, which can be regarded as the linear growth rate of $x$. Here, parameter $A$ is used to control the amplitude of the diurnal growth rate. When $A=0$, this model is reduced to the classical Lorenz63 model. By utilizing this diurnal cycle of growth rate, we can reveal the features of DPB in $x, y$, and $z$ in the Lorenz63 model. The Lorenz63 model is solved using the fourth-order Runge-Kutta scheme, with a time step of 0.005 , which is smaller than that used in Lorenz63. Generally, a smaller time step gives a more precise result.

First, we fit the Lorenz63 model with weather observations via autocorrelation. By analyzing the surface temperature data in Wisconsin and Beijing (Fig. 1a), we find the autocorrelation of the temperature roughly decays to 0.1 after 4 days in both places. In the Lorenz63 model, when $A=0$, the autocorrelation of $x$ decays to 0.1 after 128 time steps (Fig. 1b). As such, we choose $128 / 4=32$ time steps to represent one day so that one time step corresponds to $0.75 \mathrm{~h}$. Further tests show that a reduction of time step does not change our results. We integrate the model for 20 years, and analyze the outputs of the last 10 years to study the DPB.

\section{c. The first-order autoregression model}

The first-order autoregression (AR1) model has been used to study ENSO spring predictability barrier extensively (Torrence and Webster 1998; Ren et al. 2016; Liu et al. 2019). Here, we use the AR1 model to explain the timing of the DPB in the observations. The AR1 model that incorporates a diurnal cycle is as follows:

$$
X_{t+1}=a_{m} X_{t}+N_{t+1}
$$

where $X$ is temperature anomaly, and subscript $t$ is the time measured in hours. The growth rate $a_{m}$ is a model parameter, which can be obtained by regressing $X_{t+1}$ onto $X_{t}$, where the subscript $m$ is the diurnal cycle in hours. The last term, $N_{t+1}$, is random noise drawn from 24 Gaussian white noise processes of zero mean and noise variance $\sigma_{m}^{2}$. Liu et al. (2019) suggested that the seasonal cycle of growth rate controls the ENSO spring 

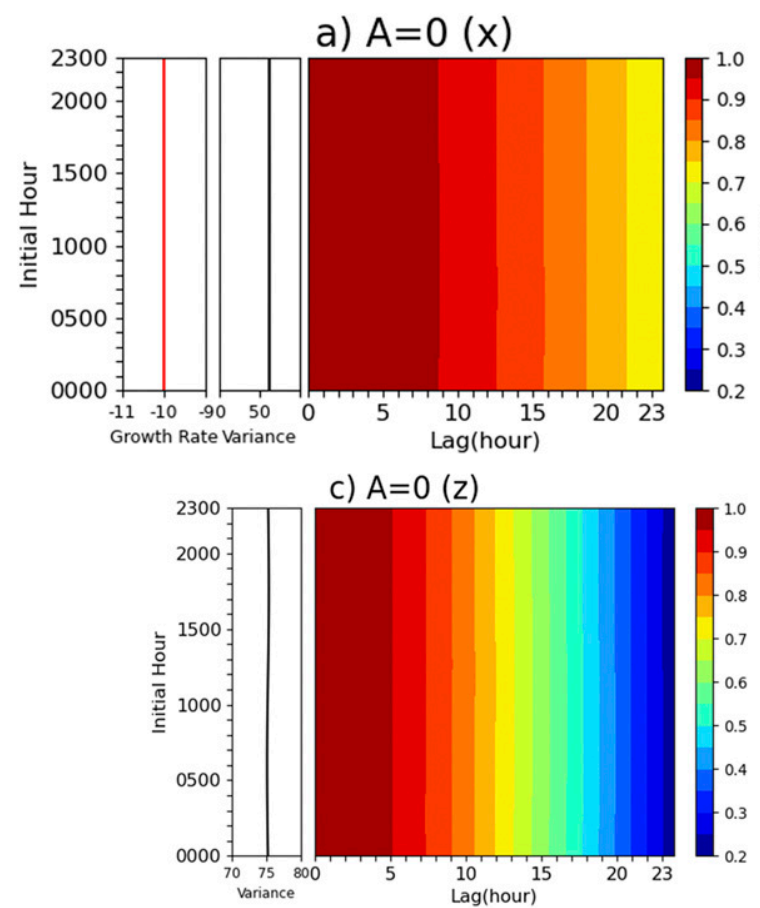

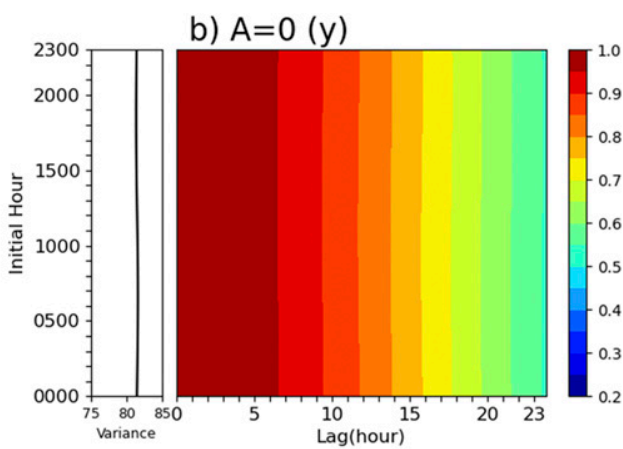

FIG. 2. The results for $A=0$ in Lorenz63 model. (a),(left) Growth rate of $x\{$ red line; $-\alpha \times[1-A \cos (\theta)]$ in Eq. (2.1) , (center) the diurnal variance (for a given initial hour, the variance of $x$ can be calculated; black line), and (right) the persistence map, or ACF $[r(m, \tau)]$ of $x$. (b),(left) Diurnal variance (black line) and (right) persistence map (right) of $y$. (c) As in (b), but for $z$. predictability barrier. We will use the diurnal growth rate $a_{m}$ and noise forcing in the real world to give a possible explanation of the timing of DPB in section 4.

\section{d. Definition of DPB strength}

ACF can be used to estimate the DPB. This is because larger autocorrelation (more persistent) in the observation tends to have higher predictability (Jin et al. 2018). ACF is a function of initial hour $m$ (ranging from 0000 to 2400) and lag hour $\tau$ (no more than 24 h; e.g., Fig. 2), which can be written as $r(m, \tau)$. Following the definition of seasonal predictability barrier strength in Liu et al. (2019), the strength of DPB is defined as follows. First, for a specific initial hour $m$, we identify $\tau_{B}(m)$ as the specific lag of maximum ACF decline (e.g., the black dots in Fig. 3a), which is calculated as the lag gradient using time step of $0.75 \mathrm{~h}$ as follows:

$$
\tau_{B}(m)=\max _{\tau}\left\{\frac{r(m, \tau-1)-r(m, \tau+1)}{2}\right\} .
$$

Then, the maximum gradient $\left[S_{B}(m)\right]$ of ACF for a specific initial hour can be defined by

$$
S_{B}(m)=\left\{\frac{r\left[m, \tau_{B}(m)-1\right]-r\left[m, \tau_{B}(m)+1\right]}{2}\right\} .
$$

Finally, the intensity $\left(S_{B 1}\right)$ of the DPB is estimated by using

$$
S_{B_{1}}=\sum_{m=0}^{32} S_{B}(m)
$$

When we perform ensemble forecasts, anomaly correlation coefficient (ACC) can be used to estimate the DPB. Similar to
$\mathrm{ACF}, \mathrm{ACC}$ is also a function of initial hour $m$ and forecast hour $\tau$, which can be written as $r \_\mathrm{ACC}(m, \tau)$. ACC is defined as the correlation coefficient between anomalies of the ensemble mean forecast and the corresponding "truth." It is an index to identify the predictability of forecasting and can be obtained as follows for a given initial hour $(m)$ :

$$
r \_\mathrm{ACC}(m, \tau)=\frac{\langle F(m, \tau), O(m, \tau)\rangle}{\sqrt{\langle F(m, \tau), F(m, \tau)\rangle\langle O(m, \tau), O(m, \tau)\rangle}},
$$

where $F(m, \tau)$ is the ensemble mean forecast anomaly at the forecast step $\tau, O(m, \tau)$ is the series of the "truth" corresponding to $F(m, \tau)$, and the angle brackets \langle\rangle denote the variance over the whole time series. We identify $\tau_{B-} \mathrm{ACC}(m)$ as the specific lag of maximum $r_{-}$ACC decline, which is calculated as the lag gradient in one forecast step $(0.75 \mathrm{~h})$ for a given initial hour $m$ as follows:

$$
\tau_{B-} \mathrm{ACC}(m)=\max _{\tau}\left\{\frac{r_{-} \mathrm{ACC}(m, \tau-1)-r_{-} \mathrm{ACC}(m, \tau+1)}{2}\right\} .
$$

The maximum gradient $\left[S_{B \_} \mathrm{ACC}(m)\right.$ of $\mathrm{ACC}$ for a specific initial hour can be defined by

$$
\begin{aligned}
& S_{B-} \mathrm{ACC}(m) \\
& =\left\{\frac{r_{-} \mathrm{ACC}\left[m, \tau_{B-} \mathrm{ACC}(m)-1\right]-r_{-} \mathrm{ACC}\left[m, \tau_{B-} \mathrm{ACC}(m)+1\right]}{2}\right\} .
\end{aligned}
$$

Finally, the intensity $\left(S_{B 1}\right.$ ACC) of the DPB is estimated using 

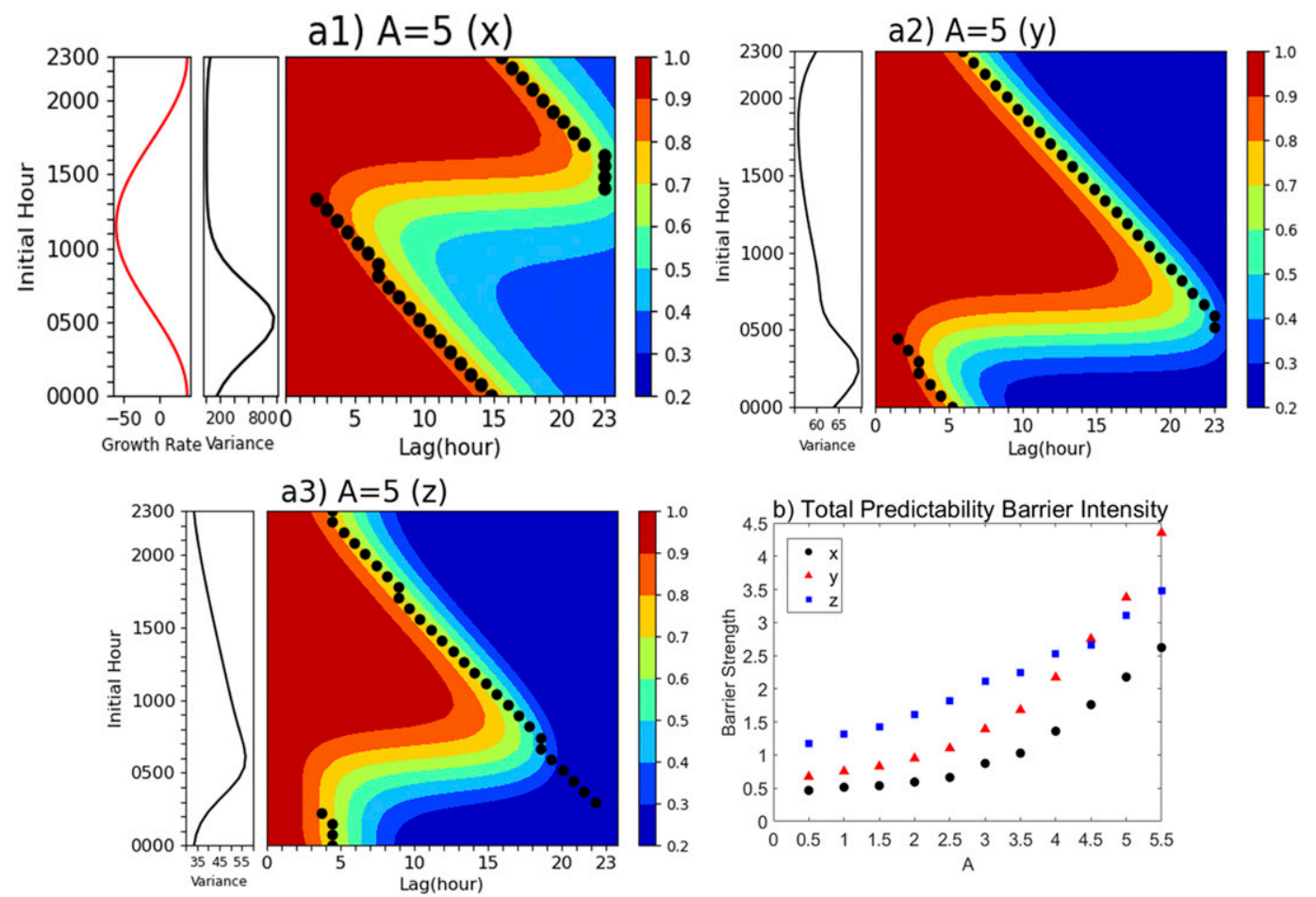

FIG. 3. As in Fig. 2, but for the $A=5$ case. [a(1)]-[a(3)]The black dots on the persistence maps mark the hours of maximum autocorrelation decline [gradient; $\tau_{B}$ in Eq. (2.6)] for different initial times. (b) The predictability barrier intensity [ $S_{B 1}$ in Eq. (2.8)] of $x$ (black dots), $y$ (red triangles), and $z$ (blue squares) for different values of (a).

$$
S_{B_{1}-} \mathrm{ACC}=\sum_{m=0}^{32} S_{B_{-}} \mathrm{ACC}(m) .
$$

\section{e. Calculation of persistence map using observation records}

The persistence map is the same as ACF map, which is a function of initial hour $(m)$ and lag hour $\tau$. For a specific calendar month (January or July) of a year, the persistence map $\left[r_{i}(m, \tau), i\right.$ ranging from 1998 to 2018 for Wisconsin station data and from 2009 to 2018 for Beijing station data] can be obtained. Second, we average the persistence map to obtain the final persistence map $[r(m, \tau)]$. That is,

$$
r(m, \tau)=\frac{1}{N} \sum_{i=\text { Min_Year }}^{i=\text { Max_Year }} r_{i}(m, \tau),
$$

where $N$ is the length of observation record. Here, we choose a specific calendar month (January or July) instead of the whole year to avoid the influence of seasonal cycle on the DPB. The average of the persistence map $\left[r_{i}(m, \tau)\right]$ is to filter out the influence of weather events, like typhoons and storms, which are highly nonlinear.

\section{DPB in Lorenz63 model}

In this section, we show that regardless of initial errors, the DPB is an intrinsic feature in the diurnal forcing Lorenz63 model.

\section{a. DPB in Lorenz63 model using ACF}

In the absence of a diurnal cycle $(A=0)$, the growth rate is constant (left panel of Fig. 2a), and there is no DPB in the
Lorenz63 model. This is obvious, as shown in the diurnal variance of $x$ (for a given initial hour $m$, the variance of $x$ can be calculated and then this diurnal variation of variance in variable $x$ can be obtained; middle panel of Fig. 2a), and the maximum autocorrelation decline occurs at the same lag from each initial hour (right panel of Fig. 2a). Note that the maximum autocorrelation decline happens in the last lag time (hour 24) for the Lorenz63 model, rather than in the initial lag time for the Langevin equation (Liu et al. 2019). This is the difference between chaotic and damped systems. Similarly, there is no DPB found in the related variables $y$ and $z$ (Figs. 2b,c).

DPB emerges when the diurnal cycle forcing strengthens in the Lorenz63 model $(A>0)$, as shown in Fig. 3. When $A=5$, the growth rate is maximum at 0000 and reaches its minimum at 1200 , as shown in the left panel of Fig. 3a(1). This diurnal cycle of growth rate suggests that $x$ is most damped at 1200 and least damped at 0000 . The variance of $x$ reaches its minimum near 1600 [middle panel of Fig. 3a(1)], a 4-h lag with the minimum growth rate [left and middle panels of Fig. 3a(1)]. This lag can be explained by the delayed response of the diurnal forcing system (Jin et al. 2019). The timing of DPB occurs near 1500 [right panel of Fig. 3a(1)]. For the initial time starting at 0000 , the maximum autocorrelation decline happens with a lag of $15 \mathrm{~h}$; for the initial time starting at 1200 , the maximum decline occurs roughly with a lag of $3 \mathrm{~h}$, which is also at 1500 , the same as that for the initial time starting at 0000 . Regardless of the initial hour, the maximum autocorrelation decline occurs at a specific hour (1500 in this case). This DPB may be caused by 

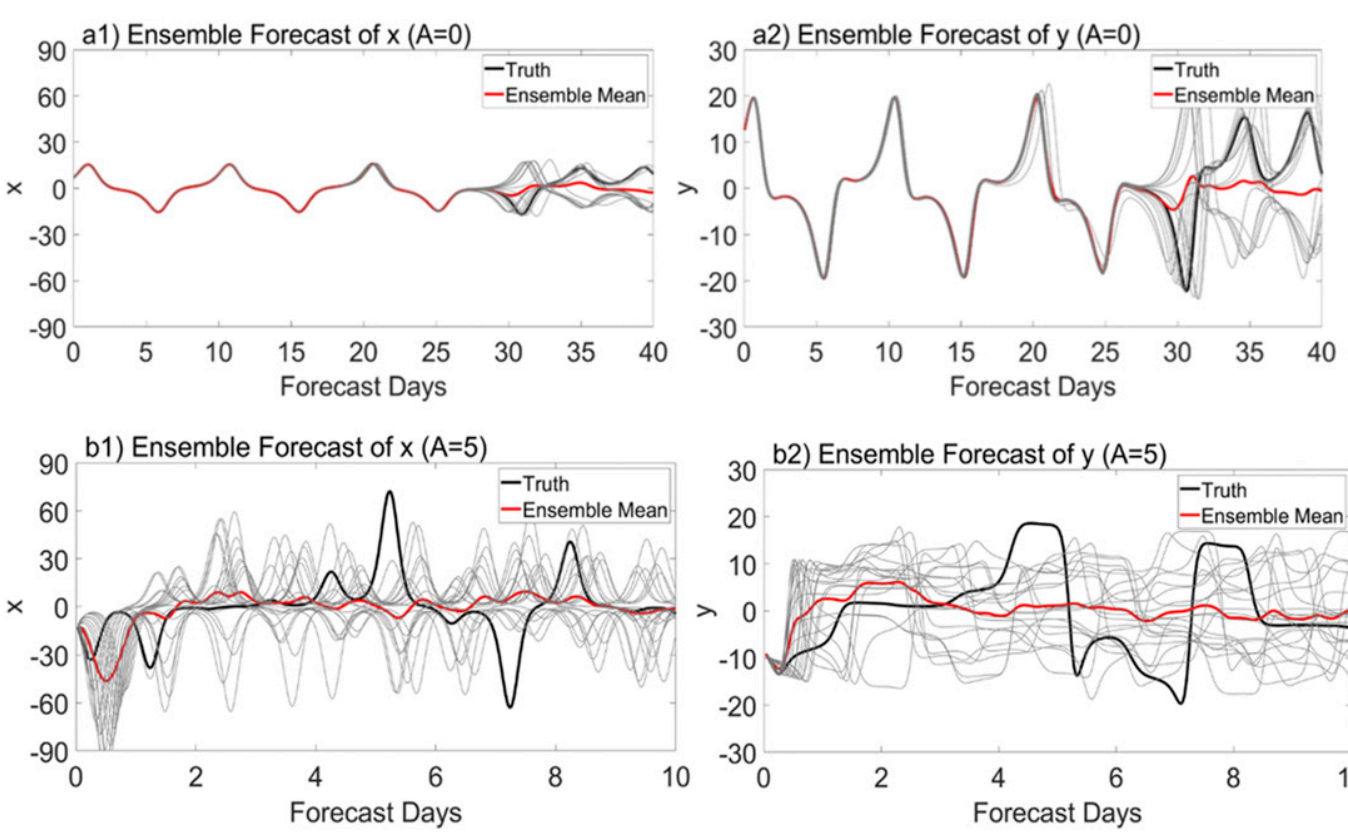

FIG. 4 [a(1)] The forecast results for $\mathrm{x}$ in the $A=0$ case. The gray line is the forecast result of every member, the red line is the result of ensemble mean, and the black line is the "truth." $[\mathrm{a}(2)] \mathrm{As}$ in $[\mathrm{a}(1)]$, but for the forecast result of $y$. [b(1)],[b(2)] As in [a(1)] and [a(2)], respectively, but for $A=5$.

the decreasing growth rate from 0000 to 1200 (Liu et al. 2019). Although the diurnal cycle is only imposed on the $x$ equation [Eq. (2.1)], $y$ and $z$ also produce distinct DPB [Figs. 3a(2),(3)] at 0600. This occurs because the diurnal cycle in $x$ affects $y$ and $z$ through both linear and nonlinear interaction terms.

The strength of the DPB is intensified by the amplitude $(A)$ of the diurnal forcing. This can be seen clearly in Fig. $3 \mathrm{~b}$. It shows the strength of DPB, which is calculated using Eq. (2.7), is a function of the strength $(A)$ of the diurnal forcing (black dots in Fig. 3b). This is consistent with the role of seasonal forcing amplitude in the linear, damped model (Liu et al. 2019). Furthermore, the strength of the DPB of $y$ and that of $z$ (red triangles and blue squares in Fig. 3b) are even stronger than that of $x$, a clear indication of the interaction among different variables.

\section{b. Ensemble forecasts in Lorenz63 model}

To show that the diurnal cycle of growth rate will also produce a DPB using ACC, regardless of initial errors, we perform ensemble weather forecasts using the Lorenz63 model in this subsection. For $A=0$, we use the $A=0$ case in section 3a (Lorenz63 model) as "truth." For each of the forecast ensemble members (20 members), we only add a small random normal perturbation (zero mean and 0.1 times standard deviation of $x$ ) to variable $x$ as the initial condition (i.e., perfect model forecasting). We do ensemble forecasts in every time step of 10 years to get sufficient forecast data. The same forecast is performed for the case of $A=5$; similarly, we set the $A=5$ case in section 3 a as "truth."

The forecast results are closer to the "truth" in the nondiurnal forcing model than in the diurnal forcing model, as shown in selected examples (Fig. 4). Without the diurnal cycle, the ensemble mean forecast (red line) matches the "truth" (black line) until the forecast length reaches about 27 days [Fig. 4a(1)]. When a strong diurnal cycle is imposed with $A=5$ [Fig. $4 \mathrm{~b}(1)$ ], the effective forecast length is reduced dramatically to smaller than one day. This shows clearly that the diurnal cycle forcing can decrease the predictability of $x$ significantly. On the other hand, although ensemble forecasts tend to bifurcate when forecast days are longer than one day, ensemble mean is still a good way to calculate ACC and evaluate the DPB. This is because no more than $24 \mathrm{~h}$ of forecasting is needed to obtain the DPB [e.g., Fig. 5a(1)]. The results are the same when we compare $y$ in the $A=0$ case with $y$ in the $A=5$ case [Fig. 4a(2) versus Fig. $4 \mathrm{~b}(2)]$. The same is also found for variable $z$ (not shown).

The forecast ACC map $\left[r_{-} \mathrm{ACC}(m, \tau)\right.$ in Eq. (2.9)] under a strong diurnal forcing $(A=5)$ shows a distinct DPB (Fig. 5). In contrast, the ACC map of $A=0$ is constant and close to 1 (not shown). The DPB of $x$ in the forecast ACC map is characterized by the maximum decline of ACC [black circles; $\tau_{B \_}$ACC in Eq. (2.10)], as shown in Fig. 5a(1). Now, the maximum ACC decline occurs at the lag of $16 \mathrm{~h}$ for the initial hour at 0000 , and at the lag of $6 \mathrm{~h}$ for the initial hour at 1000 . This suggests that the timing of this DPB occurs near 1600, which is close to the timing of DPB calculated using ACF [1500 in Fig. 3a(1)]. These results suggest that the DPB always exists in the diurnal forcing system [Fig. $3 a(1)$ versus Fig. $5 a(1)$ ], regardless of the way (ACF or ACC) used. The DPB of $x$ using ACC is also caused by the decreasing growth rate from 0000 to 1200 . Meanwhile, a strong DPB is found in both $y$ and $z$ [Figs. 5a(2),(3)], but with different timings. The DPB features in $y$ and $z$ using ACC are also close to the DPB features in $y$ and $z$ using ACF. Figure 5b 

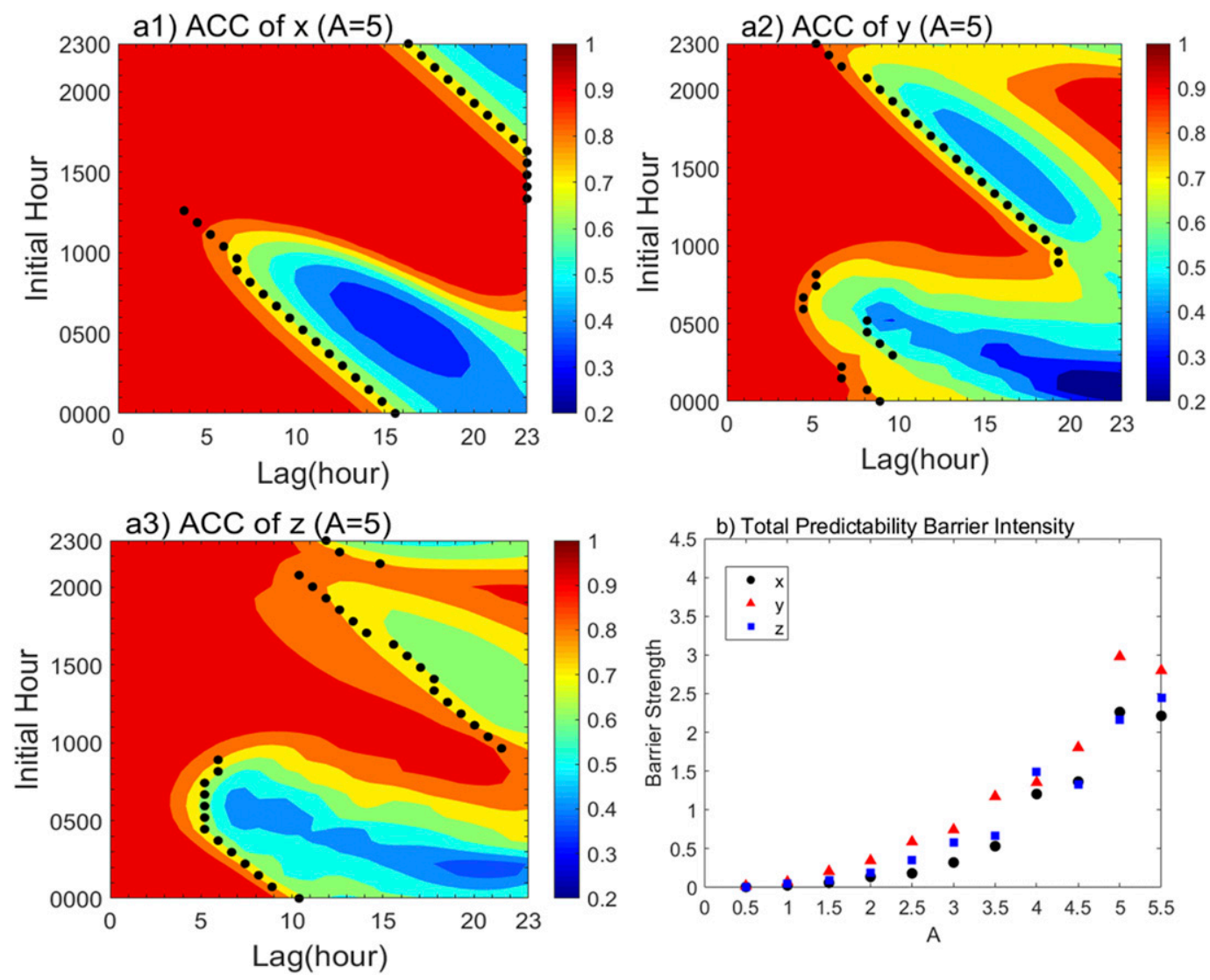

FIG. 5. The DPB (using ACC) when we perform ensemble forecasts for $A=5$. [a(1)]-[a(3)] The black dots on $r_{-}$ACC [Eq. (2.9)] mark the hours of maximum ACC decline [ $\tau_{B \_}$ACC in Eq. (2.10)] for different initial hours. [a(1)] $r \_A C C$ of $x$

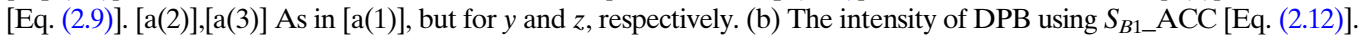

indicates that the DPB strength using ACC is positively related to the amplitude of the diurnal forcing, which is consistent with the results of DPB using ACF (Fig. 3b). Our additional experiments with different sizes of initial errors show that the DPB using ACC is robust and independent of initial errors (Fig. 6).

From the evidence presented in sections $3 a$ and $3 b$, the DPB is an intrinsic feature in the Lorenz63 model with diurnal cycle forcing. While the Lorenz equations do not correspond directly to large-scale atmospheric equations of motion, there are striking qualitative similarities with the behaviors of the largescale atmosphere, notably the existence of regime structure, distinct time scales and variations in local predictability estimates around the Lorenz attractor (Palmer 1993). As such, a DPB may exist in the surface temperature observations as a prominent diurnal cycle is present in the daily weather variability.

\section{DPB in observation records using $\mathrm{ACF}$}

In this section, we show DPB in the observation records by examining the surface temperature data in Wisconsin $\left(45.8^{\circ} \mathrm{N}, 90.0^{\circ} \mathrm{W}\right)$ and Beijing $\left(40.0^{\circ} \mathrm{N}, 116.5^{\circ} \mathrm{E}\right)$. We start with Wisconsin data.
There is a strong DPB for temperature anomalies in Wisconsin in both January and July (Fig. 7). For a specific month of the year in Wisconsin, we calculate the ACF and then average the ACF from 1998 to 2018 to obtain a persistence map

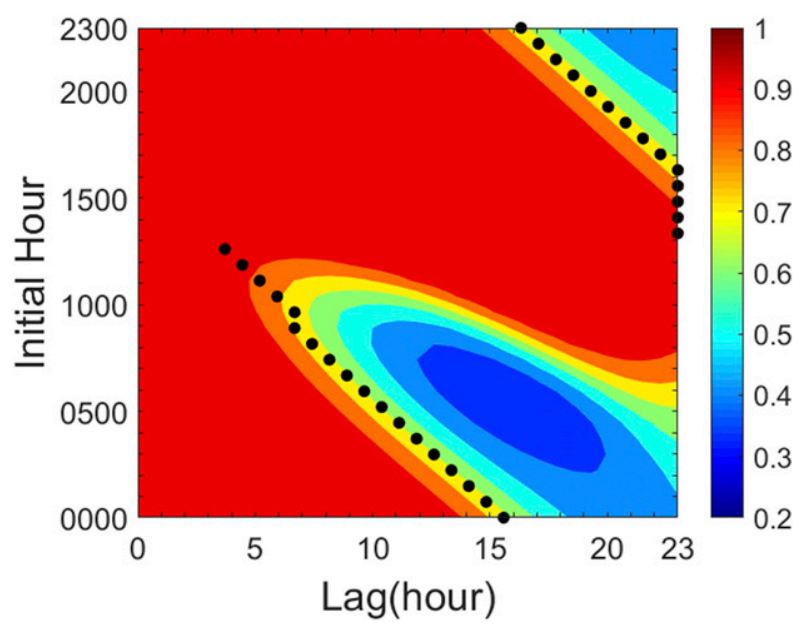

FIG. 6. As in Fig. 5[a(1)], but for a smaller initial-condition error $(0.01 \times$ the standard deviation of $x)$. 


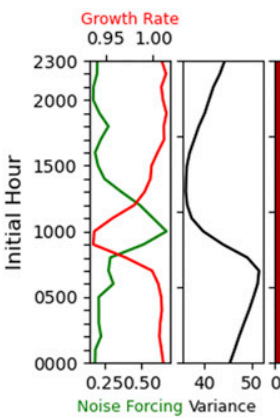

a) ACF of Wisconsin (Jan)

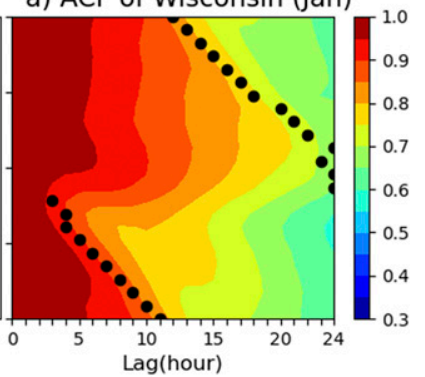

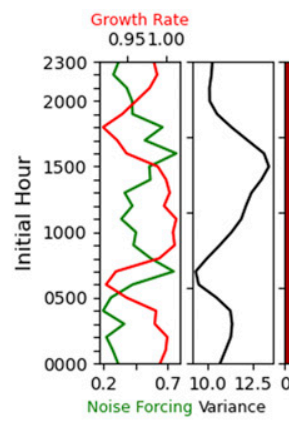

b) ACF of Wisconsin (Jul)

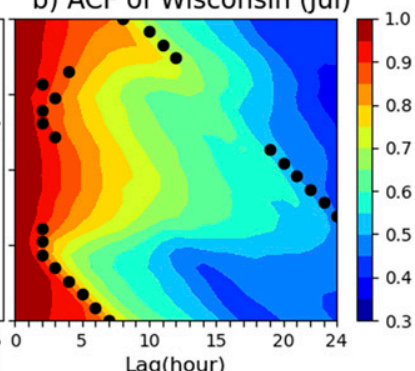

FIG. 7. DPB in Wisconsin. (a),(left) Diurnal growth rate (red line) and diurnal noise forcing (green line) derived via the AR1 model, (center) observational diurnal variance in January, and (right) the persistence map calculated using the observation data in January (section 2e). (b) As in (a), but for July.

(section 2e). Figure 7a shows a strong DPB in Wisconsin in January at 1100 (hereafter, we use local time to describe the timing of DPB in the observation). It shows that regardless of the initial hour, the predictability drops dramatically at 1100 . To give a possible explanation why the DPB occurs at 1100, we use the AR1 model (e.g., Hasselmann and Barnett 1981; Ruiz de Elvira and Lemke 1982; Torrence and Webster 1998), in which the 1-h-lag regression coefficient is regarded as the growth rate and the residual is the noise variance [Eq. (2.5), and appendix $\mathrm{C}$ of Liu et al. 2019]. The AR1 model indicates that the growth rate of surface temperature in Wisconsin is the lowest near 1000 when noise forcing reaches its maximum (left panel of Fig. 7a). Following Liu et al. (2019), the decreasing growth rate and increasing noise forcing may lead to a DPB in Wisconsin in July. A DPB feature is also seen in the July data from the Wisconsin site, which is depicted in the right panel of Fig. $7 \mathrm{~b}$; and its phase is locked to 0700 . The growth rate is low at 0700, while the noise forcing reaches its maximum, which may lead to the DPB feature.

The DPB feature in Beijing is not obvious in January, but is distinct in July (Fig. 8). In January, a clear DPB feature appears from 0000 to 1000 (Fig. 8a), but is not apparent afterward. The difference between the Wisconsin and Beijing results indicates that the DPB feature varies from area to area. While the DPB features are not consistent between the Beijing and Wisconsin temperatures in January, they both exhibit a clear DPB near 0700 in July (Fig. 7b versus Fig. 8b). In the Beijing data, we find a strong increasing noise forcing and a weak decreasing growth rate, which may lead to the DPB in Beijing in July.

In this section, we present the distinct DPB in the observation records (i.e., surface temperature) using ACF. The DPB discovered here causes the predictability of surface temperature to drop dramatically at a specific time of day, regardless of the initial hour.

\section{Summary and discussion}

In this paper, we expanded the theory of Liu et al. (2019), which explored the ENSO spring predictability barrier over the annual time scale and applied it to the DPB over the daily time scale. By employing the Langevin equation with seasonal coefficients, Liu et al. (2019) indicated that a strong seasonal growth rate is the necessary condition to form the ENSO spring predictability barrier. Based on their findings, we examined whether or not this theory applies to the variables with a strong diurnal cycle. Using the Lorenz63 model, we find that this linear, damped theory fits well with the chaotic system as the diurnal forcing plays a prominent role in causing a DPB on the daily time scale. In particular, the DPB is an intrinsic feature in a diurnally forced chaotic system. The DPB becomes stronger when the amplitude of the diurnal forcing becomes larger. We also examined the time series of surface temperature, which has a strong diurnal cycle, in the observation records of two stations, and revealed the existence of DPB using
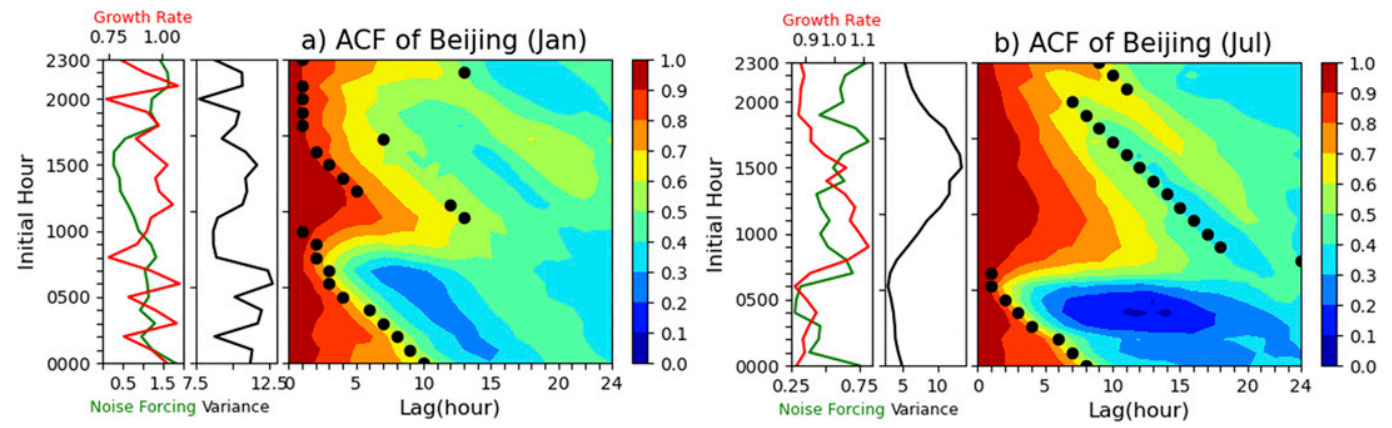

FIG. 8. As in Fig. 7, but for Beijing. 
$\mathrm{ACF}$ at both sites. Observed surface temperature data at both Wisconsin and Beijing sites were found to have a strong DPB. The predictability of surface temperature is more likely to be relatively low during the specific hour in a day, which is caused by the diurnal forcing in the system. Note that the maximum predictability drop is compared with the loss of predictability for other lead times. The DPB does not mean that the predictability is absolutely low. On the other hand, the weather is also influenced by many weather events, such as typhoons, storms, or heavy rain, which are highly nonlinear (Lorenz 1969). During these events, the DPB is meaningless.

More studies should be carried out for DPB in the observation records and its regional variation. In this paper, we use two surface observation sites to see whether a DPB exists in the observation record. Although these two sites are both near $40.0^{\circ} \mathrm{N}$, they show different DPB features in January. Additional research should be conducted to assess DPB in different geographic locations, from land to sea, and to assess DPB above the surface. Meanwhile, more studies should be carried out to check whether a DPB exists in the ensemble forecast using the state-of-art weather prediction model. We also want to note that some other variables may also have DPB features. As suggested by the Lorenz63 model, variables $y$ and $z$ can have strong DPB features as they are related to $x$. Accordingly, some variables (e.g., sea level pressure) that do not have a strong diurnal cycle should also be examined.

The expansion of the predictability barrier theory to a chaotic system also gives us a new insight into the ENSO spring predictability barrier. Since the theory of Liu et al. (2019) derived from a linear stochastic model can be used to explain the DPB in the diurnally varying Lorenz63 model, it may provide an explanation of predictability barrier phenomenon, regardless of a damped system or a chaotic system.

Acknowledgments. We thank Prof. A. Desai from the University of Wisconsin-Madison for the Wisconsin time series. We also thank the editor and three anonymous reviewers for their helpful comments on this research. This study is supported by Chinese MOST (2017YFA0603801), China NSF (NSFC41630527), and U.S. NSF (AGS-1656907).

Data availability statement. Wisconsin station data can be downloaded online http://co2.aos.wisc.edu/data/cheas/wcreek/ flux/current/. Beijing Capital International Airport station data can be accessed at https://www.ncei.noaa.gov/data/global-hourly/ access/. The Lorenz63 model (including the forecast data) used in this paper can be found online: http://doi.org/ 10.5281/zenodo.4441291.

\section{APPENDIX}

\section{Null Hypothesis Relative to Diurnal Predictability Barrier}

The Langevin equation (Hasselmann 1976) with periodic forcing can be written as follows:

$$
\frac{d T}{d t}+b(t) T=N(t)
$$

Here, we assume that $b(t)$ is periodic forcing and can be written as

$$
b(t)=b_{0}[1-A \cos (\omega t)]
$$

where $A$ is the amplitude of the periodic forcing, $b_{0}$ is the periodic mean, $T$ is a variable, and $N$ is the noise forcing. The autocorrelation function of $T[r(m, \tau)]$, which is the function of initial time $(m)$ and lag time $(\tau)$, can be derived as follows:

$$
r(m, \tau) \approx e^{-b_{0} \tau}\left[1+\frac{A B^{2}}{\sqrt{1+B^{2}}} \sin \left(\frac{\omega \tau}{2}\right) \cos \left(\omega m+\frac{\omega \tau}{2}-\theta_{1}\right)\right],
$$

where $B=2 b_{0} / \omega$, and $\theta_{1}=\arcsin \left(1 / \sqrt{1+B^{2}}\right)$.

For a very weak damping system, that is, $B \ll 1$ (when $B$ gets larger, the numerical solution also fits well with the analytical solution; Liu et al. 2019), the autocorrelation function gradient at every lag step for a given initial time $(m)$ can be derived as follows:

$$
\partial_{\tau} r(m, \tau) \approx-b_{0} e^{-b_{0} \tau}\left[1-\frac{A B}{\sqrt{1+B^{2}}} \cos \left(\omega m+\omega \tau-\theta_{1}\right)\right] .
$$

Based on this gradient function [Eq. (A4)], the maximum gradient at the specific lag time $\left[\tau_{B}\right.$; also see Eq. (2.6)] for every initial time $(\mathrm{m})$ can be found as follows:

$$
\partial_{\tau \tau} r(m, \tau) \approx b_{0}^{2} e^{-b_{0} \tau}\left[1-2 A \cos \left(\omega m+\omega \tau_{B}-2 \theta_{1}\right)\right]=0 .
$$

Therefore, a predictability barrier occurs in the fixed phase:

$$
\omega\left(m+\tau_{B}\right)=\arccos \left(\frac{1}{2 A}\right)+2 \theta_{1} .
$$

According to Eq. (A6), for different initial time $m$, the maximum gradient lag $\left(\tau_{B}\right)$ is different. However, $m+\tau_{B}$ is fixed ( $A$ or $B$ is fixed for a specific system). This indicates the feature of predictability barrier: maximum drop of predictability at a particular time of the period, regardless of the initial time. This feature is not obvious as the timing of predictability barrier is fixed for different initial times in a periodically varying system.

Therefore, when $\omega$ in Eq. (A2) is set to the annual frequency, this analytical solution can be used to explain the ENSO predictability barrier because the seasonal forcing amplitude in the tropical Pacific is strong (Liu et al. 2019). When $\omega$ is set to the diurnal frequency, it may also explain the diurnal predictability barrier because the diurnal cycle is also strong.

\section{REFERENCES}

Hasselmann, K. 1976: Stochastic climate models: Part I. Theory. Tellus, 28, 473-485, https://doi.org/10.1111/J.21533490.1976.TB00696.X.

— systems with periodic statistics. J. Atmos. Sci., 38, 2275-2283, https://doi.org/10.1175/1520-0469(1981)038<2275:TOLPFS > 2.0.CO;2.

Jin, Y. X. Rong, and Z. Liu, 2018: Potential predictability and forecast skill in ensemble climate forecast: A skill-persistence rule. Climate Dyn., 51, 2725-2742, https://doi.org/10.1007/ s00382-017-4040-z. 
_ Z. Liu, and X. Rong, 2019: General seasonal phase-locking of variance and persistence: Application to tropical Pacific, North Pacific and global ocean. Climate Dyn., 53, 2825-2842, https://doi.org/10.1007/s00382-019-04659-7.

Levine, A. F., and M. J. McPhaden, 2015: The annual cycle in ENSO growth rate as a cause of the spring predictability barrier. Geophys. Res. Lett., 42, 5034-5041, https://doi.org/ 10.1002/2015GL064309.

Liu, Z., Y. Jin, and X. Rong, 2019: A theory for the seasonal predictability barrier: Threshold, timing, and intensity. J. Climate, 32, 423-443, https://doi.org/10.1175/JCLI-D-180383.1.

Lorenz, E. N., 1963: Deterministic nonperiodic flow. J. Atmos. Sci., 20, 130-141, https://doi.org/10.1175/1520-0469(1963) $020<0130: \mathrm{DNF}>2.0 . \mathrm{CO} ; 2$.

_ - 1969: The predictability of a flow which possesses many scales of motion. Tellus, 21, 289-307, https://doi.org/10.3402/ tellusa.v21i3.10086.

Orrell, D., 2002: Role of the metric in forecast error growth: How chaotic is the weather? Tellus, $\mathbf{5 4 A}, 350-362$, https://doi.org/ 10.3402/tellusa.v54i4.12159.
Palmer, T. N., 1993: Extended-range atmospheric prediction and the Lorenz model. Bull. Amer. Meteor. Soc., 74, 49-66, https://doi.org/ 10.1175/1520-0477(1993)074<0049:ERAPAT>2.0.CO;2.

Ren, H. L., F. F. Jin, B. Tian, and A. A. Scaife, 2016: Distinct persistence barriers in two types of ENSO. Geophys. Res. Lett., 43, 10 973-10 979, https://doi.org/10.1002/2016GL071015.

Ruiz de Elvira, A., and P. Lemke, 1982: A Langevin equation for stochastic climate models with periodic feedback and forcing variance. Tellus, 34, 313-320, https://doi.org/10.3402/tellusa.v34i4.10818.

Torrence, C., and P. J. Webster, 1998: The annual cycle of persistence in the El Niño/Southern Oscillation. Quart. J. Roy. Meteor. Soc., 124, 1985-2004, https://doi.org/10.1002/qj.49712455010.

Troup, A., 1965: The 'Southern Oscillation.' Quart. J. Roy. Meteor. Soc., 91, 490-506, https://doi.org/10.1002/qj.49709139009.

Walker, G. T., and E. W. Bliss, 1932: World weather V. Mem. Roy. Meteor. Soc, 4, 53-84.

Webster, P. J., and S. Yang, 1992: Monsoon and ENSO: Selectively interactive systems. Quart. J. Roy. Meteor. Soc., 118, 877-926, https://doi.org/10.1002/qj.49711850705.

Wright, P. B., 1979: Persistence of rainfall anomalies in the central Pacific. Nature, 277, 371-374, https://doi.org/10.1038/277371a0. 